\title{
Drastic Attenuation of Pseudomonas aeruginosa Pathogenicity in a Holoxenic Mouse Experimental Model Induced by Subinhibitory Concentrations of Phenyllactic acid (PLA)
}

\author{
Mariana-Carmen Chifiriuc ${ }^{1}{ }^{*}$, Lazar Veronica ${ }^{1}$, Olguta Dracea ${ }^{2}$, Lia-Mara Ditu ${ }^{1}$, \\ Diana Smarandache ${ }^{1}$, Marcela Bucur ${ }^{1}$, Cristina Larion ${ }^{2}$, Ramona Cernat ${ }^{1}$ and \\ Elena Sasarman ${ }^{1}$
}

1 Department of Microbiology, Faculty of Biology, University of Bucharest, Aleea Portocalelor 1-3, Bucharest 060101, Romania; E-mails: ciprian.alexandru@ gmail.com (Lazar Veronica); lia_mara_d@yahoo.com (Lia-Mara Ditu); diana_smarandache@yahoo.com (Diana Smarandache); bmarcelica@yahoo.com (Marcela Bucur); ramon_70009@yahoo.com (Ramona Cernat); vibrio@cantacuzino.ro (Elena Sasarman)

2 Cantacuzino Institute, Spl. Independentei 103, P.O.B.- 1-525, Bucharest, Romania; E-mails: vibrio@cantacuzino.ro (Olguta Dracea); larioncristina@yahoo.com (Cristina Larion)

* Author to whom correspondence should be addressed; E-mail: carmen_balotescu@ yahoo.com Received: 29 January 2007; in Revised Form: 25 June 2007 / Accepted: 26 June 2007 / Published: 2 July 2007

\begin{abstract}
The discovery of communication systems regulating bacterial virulence has afforded a novel opportunity to control infectious bacteria without interfering with growth. In this paper we describe the effect of subinhibitory concentrations of phenyllactic acid (PLA) on the pathogenicity of Pseudomonas aeruginosa in mice. The animals were inoculated by oral (p.o.), intranasal (i.n.), intravenous (i.v.) and intraperitoneal (i.p.) routes with $P$. aeruginoasa wild and PLA-treated cultures. The mice were followed up during 16 days after infection and the body weight, mortality and morbidity rate were measured every day. The microbial charge was studied by viable cell counts in lungs, spleen, intestinal mucosa and blood. The mice batches infected with wild $P$. aeruginosa bacterial cultures exhibited high mortality rates (100\% after i.v. and i.p. route) and very high cell counts in blood, lungs, intestine and spleen. In contrast, the animal batches infected with PLA treated bacterial cultures exhibited good survival rates ( $0 \%$ mortality)
\end{abstract}


and the viable cell counts in the internal organs revealed with one exception the complete abolition of the invasive capacity of the tested strains. In this study, using a mouse infection model we show that $D$-3-phenyllactic acid (PLA) can act as a potent antagonist of Pseudomonas (P.) aeruginosa pathogenicity, without interfering with the bacterial growth, as demonstrated by the improvement of the survival rates as well as the clearance of bacterial strains from the body.

Keywords: Phenyllactic acid, Pseudomonas aeruginosa, in vivo holoxenic mouse model, virulence attenuation

\section{Introduction}

Upon contact with the host cells many pathogens use intercellular communication mechanisms for the regulation of virulence factors expression. One of the newest strategies for the prevention and treatment of bacterial infections is the inhibition of cell-to-cell signalling by inhibitors which do not interfere with the microbial growth [1]. Pseudomonas aeruginosa is an increasingly prevalent opportunistic pathogen and is the most common Gram-negative bacterium found in nosocomial and life threatening infections of immunocompromised patients [2]. P. aeruginosa, besides its natural resistance to a large number of antibiotics effective against other Gram-negative bacilli such as the A group penicillins, 1st-3rd generation cephalosporins, tetracyclins and trimetoprim, has developed acquired resistance to the so-called "antipseudomonals": carboxypenicillins (ticarcillin, carbenicillin, 25-30 \% resistance), acyl-ureidopenicillins (cephtazidime, cephsulodin), carbapenems (imipenem, $20-25 \%)$, gentamycin (47\%) and ciprofloxacin (35-40\%).

The intrinsic resistance of Pseudomonas cells towards antimicrobial agents is further increased when the bacteria is growing in biofilms. In fact, biofilms are known for their high resistance to antimicrobial agents, thus explaining their implication in the etiology of infectious diseases, with an incidence as high as $65 \%$ [2]. Human $P$. aeruginosa infections involving bacterial adherence and biofilm development on medical devices are very difficult to treat and eradicate, due to their increased resistance to the anti-infective host defense [3]. The attempts made until present to attenuate bacterial pathogenesis due to $P$. aeruginosa by interfering with bacterial cell to cell communication, without interfering with growth have used natural antagonists produced by superior organisms (furanones) or chemically synthesized [6].

The present study was aimed at attenuating the pathogenicity in $P$. aeruginosa by using subinhibitory concentrations of phenyl lactic acid (PLA). Our approach is based on the fact that this compound was found in the cell-free cultures of Lactobacillus probiotic strains and its antibacterial activity was already demonstrated for Gram-positive microorganisms [7]. Our previous studies with cell-free probiotic cultures liquids have also demonstrated that the probiotic bacteria secrete soluble factors interfering with the pathogenic bacteria and host cell signaling mechanisms, in the first case by inducing changes in the expression of surface molecules and consequently, the shift of the adherence pattern from aggregative to the diffuse one, and the stimulation of endocytic processes of cultured cells, respectively [8]. 


\section{Results and Discussion}

The ability of pathogenic bacteria to survive in different environments illustrates the importance of understanding the regulation of bacterial virulence genes and those implicated in the detection of the environmental signals for the elaboration of adequate anti-infective strategies [9]. The success of any pathogen in the colonization of a sensitive host and the development of an infectious process is dependent on its ability to sense its environment and to modulate the expression of the gene encoding factors required for the establishment and adaptation to the new habitat [10].

Table 1. Mortality and body weight dynamics in animals infected with P. aeruginosa wild cultures.

\begin{tabular}{|c|c|c|c|c|c|c|c|c|c|c|}
\hline \multirow{2}{*}{ No. } & \multirow{2}{*}{$\begin{array}{l}\text { Bacterial strain/ } \\
\text { Inoculation mode }\end{array}$} & \multicolumn{3}{|c|}{ Day 0} & \multicolumn{3}{|c|}{ Day 1} & \multicolumn{3}{|c|}{ Day 2} \\
\hline & & no. & $\mathrm{g} / \mathrm{b}$ & $\mathrm{g} / \mathrm{m}$ & no. & $g / b$ & $\mathrm{~g} / \mathrm{m}$ & no. & $g / b$ & $\mathrm{~g} / \mathrm{m}$ \\
\hline 1. & $\begin{array}{l}\text { P. aeruginosa } \\
247 / 1464 \text { i.p. }\end{array}$ & 5 & 107 & 26.75 & 2 & 20 & 10 & 1 & 23 & 23 \\
\hline 2. & $\begin{array}{l}\text { P. aeruginosa } \\
247 / 1464 \text { i.v. }\end{array}$ & 5 & 102 & 25.5 & - & - & - & - & - & - \\
\hline 3. & $\begin{array}{l}P . \text { aeruginosa } \\
\text { 247/1464 i.n. }\end{array}$ & 5 & 92 & 23 & 4 & 90 & 22.5 & 4 & 90 & 22.5 \\
\hline 4. & $\begin{array}{l}\text { P. aeruginosa } 492 \\
\text { i.p. }\end{array}$ & 5 & 110 & 27.5 & - & - & - & - & - & - \\
\hline 5. & $\begin{array}{l}\text { P. aeruginosa } 492 \\
\text { i.v. }\end{array}$ & 5 & 99 & 24.75 & 3 & 55 & 18.53 & - & - & - \\
\hline 6. & $\begin{array}{l}\text { P. aeruginosa } 492 \\
\text { i.n. }\end{array}$ & 5 & 92 & 23 & 4 & 90 & 22.5 & 4 & 96 & 24 \\
\hline 7. & $\begin{array}{l}\text { P. aeruginosa } 492 \\
\text { p.o. }\end{array}$ & 5 & 94 & 23.5 & 4 & 90 & 22.5 & 4 & 94 & 23.5 \\
\hline 8. & $\begin{array}{l}\text { P. aeruginosa } 37 \\
\text { i.p. }\end{array}$ & 5 & 95 & 23.75 & - & - & - & - & - & -- \\
\hline 9. & $\begin{array}{l}P . \text { aeruginosa } 37 \\
\text { i.v. }\end{array}$ & 5 & 92 & 23 & - & - & - & - & - & - \\
\hline 10. & $\begin{array}{l}P . \text { aeruginosa } 37 \\
\text { i.n. }\end{array}$ & 5 & 92 & 23 & 4 & 89 & 22.25 & 4 & 96 & 24 \\
\hline 11. & $\begin{array}{l}P . \text { aeruginosa } 37 \\
\text { p.o. }\end{array}$ & 5 & 97 & 24.25 & 4 & 95 & 23.75 & 4 & 106 & 26.5 \\
\hline 12. & $\begin{array}{l}\text { Ps. aeruginosa } \\
1489 \text { i.p. }\end{array}$ & 5 & 88 & 22 & - & - & - & - & - & - \\
\hline 13. & $\begin{array}{l}\text { P. aeruginosa } 1489 \\
\text { i.v. }\end{array}$ & 5 & 77 & 19.25 & - & - & - & - & - & - \\
\hline 14. & $\begin{array}{l}\text { P. aeruginosa } 1489 \\
\text { i.n. }\end{array}$ & 5 & 107 & 26.75 & 4 & 78 & 19.5 & 4 & 86 & 21.5 \\
\hline 15. & $\begin{array}{l}\text { P. aeruginosa } 1489 \\
\text { p.o. }\end{array}$ & 5 & 82 & 20.5 & 4 & 87 & 21.75 & 4 & 95 & 23.75 \\
\hline 16. & Control & 5 & 74 & 18.5 & 4 & 76 & 19 & 4 & 76 & 19 \\
\hline
\end{tabular}


Table 1. Cont.

\begin{tabular}{|c|c|c|c|c|c|c|c|c|c|c|}
\hline \multirow[b]{2}{*}{ No. } & \multirow{2}{*}{$\begin{array}{l}\text { Bacterial strain/ } \\
\text { Inoculation } \\
\text { mode }\end{array}$} & \multicolumn{3}{|c|}{ Day 7} & \multicolumn{3}{|c|}{ Day 9} & \multicolumn{3}{|c|}{ Day 16} \\
\hline & & no. & $\mathrm{g} / \mathrm{b}$ & $\mathrm{g} / \mathrm{m}$ & no. & $\mathrm{g} / \mathrm{b}$ & $\mathrm{g} / \mathrm{m}$ & no. & $\mathbf{g} / \mathbf{b}$ & $\mathrm{g} / \mathrm{m}$ \\
\hline 1. & $\begin{array}{l}\text { P. aeruginosa } \\
\text { 247/1464 i.p. }\end{array}$ & 1 & 24 & 24 & 1 & 21 & 21 & 1 & 28 & 28 \\
\hline 2. & $\begin{array}{l}\text { P. aeruginosa } \\
247 / 1464 \text { i.v. }\end{array}$ & - & - & - & - & - & - & - & - & - \\
\hline 3. & $\begin{array}{c}P . \text { aeruginosa } \\
\text { 247/1464 i.n. }\end{array}$ & 4 & 95 & 23.75 & 4 & 95 & 23.75 & 4 & 107 & 26.75 \\
\hline 4. & $\begin{array}{c}P . \text { aeruginosa } \\
\text { 247/1464 p.o. }\end{array}$ & 4 & 93 & 23.25 & 4 & 100 & 25 & 4 & 112 & 28 \\
\hline 5. & $\begin{array}{l}\text { P. aeruginosa } \\
492 \text { i.p. }\end{array}$ & - & - & - & - & - & - & - & - & - \\
\hline 6. & $\begin{array}{l}\text { P. aeruginosa } \\
492 \text { i.v. }\end{array}$ & - & - & - & - & - & - & - & - & - \\
\hline 7. & $\begin{array}{l}\text { P. aeruginosa } \\
492 \text { i.n. }\end{array}$ & 4 & 117 & 29.25 & 4 & 119 & 29.75 & 4 & 135 & 33.75 \\
\hline 8. & $\begin{array}{l}\text { P. aeruginosa } \\
492 \text { p.o. }\end{array}$ & 4 & 105 & 26.25 & 4 & 105 & 26.25 & 4 & 117 & 29.25 \\
\hline 9. & $\begin{array}{l}\text { P. } \text { aeruginosa } \\
37 \quad \text { i.p. }\end{array}$ & - & - & - & - & - & - & - & - & - \\
\hline 10. & $\begin{array}{l}\text { P. } \text { aeruginosa } \\
37 \quad \text { i.v. }\end{array}$ & - & - & - & - & - & - & - & - & - \\
\hline 11. & $\begin{array}{lc}\text { Ps. } & \text { aeruginosa } \\
37 & \text { i.n. }\end{array}$ & 4 & 116 & 29 & 4 & 119 & 29.75 & 4 & 121 & 30.25 \\
\hline 12. & $\begin{array}{l}\text { P. } \text { aeruginosa } \\
37 \text { p.o. }\end{array}$ & 4 & 118 & 29.5 & 4 & 123 & 30.75 & 4 & 122 & 30.5 \\
\hline 13. & $\begin{array}{l}P . \text { aeruginosa } \\
1489 \text { i.p. }\end{array}$ & - & - & - & - & - & - & - & - & - \\
\hline 14. & $\begin{array}{l}\text { P. aeruginosa } \\
1489 \text { i.v. }\end{array}$ & - & - & - & - & - & - & - & - & - \\
\hline 15. & $\begin{array}{l}\text { P. aeruginosa } \\
1489 \text { i.n. }\end{array}$ & 4 & 118 & 27 & 4 & 112 & 28 & 4 & 130 & 32.5 \\
\hline 16. & $\begin{array}{l}\text { P. aeruginosa } \\
1489 \text { p.o. }\end{array}$ & 4 & 100 & 25 & 4 & 120 & 30 & 4 & 138 & 34.5 \\
\hline 17. & Control & 4 & 77 & 19.25 & 4 & 79 & 19.75 & 4 & 100 & 25 \\
\hline
\end{tabular}

no. = number of mice/batch; $\mathrm{g} / \mathrm{b}=$ gram $/ \mathrm{batch} ; \mathrm{g} / \mathrm{m}=\mathrm{gram} / \mathrm{mouse} ;-=$ animals died during the experiment 
Table 2. Mortality and body weight dynamics in animals infected with PLA treated $P$. aeruginosa cultures.

\begin{tabular}{|c|c|c|c|c|c|c|c|c|c|c|}
\hline \multirow[b]{2}{*}{ No. } & \multirow{2}{*}{$\begin{array}{l}\text { Bacterial strain/ } \\
\text { Inoculation } \\
\text { mode }\end{array}$} & \multicolumn{3}{|c|}{ Day 0} & \multicolumn{3}{|c|}{ Day 1} & \multicolumn{3}{|c|}{ Day 2} \\
\hline & & no. & $\mathbf{g} / \mathbf{b}$ & $\mathrm{g} / \mathrm{m}$ & no. & $\mathrm{g} / \mathrm{b}$ & $\mathbf{g} / \mathbf{m}$ & no. & $\mathbf{g} / \mathbf{b}$ & $\mathrm{g} / \mathrm{m}$ \\
\hline 1. & $\begin{array}{l}P . \text { aeruginosa } \\
\text { 247/1464 i.p. }\end{array}$ & 5 & 122 & 30.5 & 4 & 118 & 29.5 & 4 & 120 & 30 \\
\hline 2. & $\begin{array}{l}P . \text { aeruginosa } \\
247 / 1464 \text { i.v. }\end{array}$ & 5 & 120 & 30 & 4 & 127 & 31.75 & 4 & 132 & 33 \\
\hline 3. & $\begin{array}{l}\text { P. aeruginosa } \\
\text { 247/1464 i.n. }\end{array}$ & 5 & 130 & 32.5 & 4 & 135 & 33.75 & 4 & 122 & 30.5 \\
\hline 4. & $\begin{array}{l}\text { P. aeruginosa } \\
\text { 247/1464 p.o. }\end{array}$ & 5 & 130 & 32.5 & 4 & 119 & 29.75 & 4 & 140 & 35 \\
\hline 5. & $\begin{array}{l}P \text {. aeruginosa } \\
492 \text { i.p. }\end{array}$ & 5 & 120 & 30 & 4 & 117 & 29.25 & 4 & 120 & 30 \\
\hline 6. & $\begin{array}{l}\text { P. aeruginosa } \\
492 \text { i.v. }\end{array}$ & 5 & 115 & 28.75 & 4 & 119 & 29.75 & 4 & 120 & 30 \\
\hline 7. & $\begin{array}{l}\text { P. aeruginosa } \\
492 \text { i.n. }\end{array}$ & 5 & 115 & 28.75 & 4 & 128 & 32 & 4 & 133 & 33,25 \\
\hline 8. & $\begin{array}{l}\text { P. aeruginosa } \\
492 \text { p.o. }\end{array}$ & 5 & 120 & 30 & 4 & 123 & 30.75 & 4 & 124 & 31 \\
\hline 9. & $\begin{array}{l}\text { P. aeruginosa } \\
37 \quad \text { i.p. } \\
\end{array}$ & 5 & 120 & 30 & 4 & 116 & 29 & 4 & 118 & 29.5 \\
\hline 10. & $\begin{array}{l}\text { P. } \text { aeruginosa } \\
37 \quad \text { i.v. } \\
\end{array}$ & 5 & 120 & 30 & 4 & 124 & 31 & 4 & 122 & 30.5 \\
\hline 11. & $\begin{array}{lc}\text { Ps. } & \text { aeruginosa } \\
37 & \text { i.n. } \\
\end{array}$ & 5 & 115 & 28.75 & 4 & 120 & 30 & 4 & 121 & 30.25 \\
\hline 12. & $\begin{array}{l}\text { P. aeruginosa } \\
37 \quad \text { p.o. }\end{array}$ & 5 & 120 & 30 & 4 & 122 & 30.5 & 4 & 122 & 30.5 \\
\hline 13. & $\begin{array}{l}\text { P. aeruginosa } \\
1489 \text { i.p. }\end{array}$ & 5 & 110 & 27.5 & 4 & 106 & 26.5 & 3 & 70 & 23.33 \\
\hline 14. & $\begin{array}{l}\text { P. aeruginosa } \\
1489 \text { i.v. }\end{array}$ & 5 & 130 & 32.5 & 4 & 120 & 30 & 4 & 130 & 32.5 \\
\hline 15. & $\begin{array}{l}P . \text { aeruginosa } \\
1489 \text { i.n. }\end{array}$ & 5 & 120 & 30 & 4 & 117 & 29.25 & 4 & 119 & 29.75 \\
\hline 16. & $\begin{array}{l}\text { P. aeruginosa } \\
1489 \text { p.o. }\end{array}$ & 5 & 120 & 30 & 4 & 110 & 27.25 & 4 & 120 & 30 \\
\hline 17. & Control & 5 & 130 & 26 & 5 & 132 & 26.4 & 5 & 122 & 30.5 \\
\hline
\end{tabular}

no. $=$ number of mice $/ \mathrm{batch} ; \mathrm{g} / \mathrm{b}=\mathrm{gram} / \mathrm{batch} ; \mathrm{g} / \mathrm{m}=\mathrm{gram} / \mathrm{mouse} ;-=$ animals died during the experiment 
Table 2. Cont.

\begin{tabular}{|c|c|c|c|c|c|c|c|c|c|c|}
\hline \multirow[b]{2}{*}{ No. } & \multirow{2}{*}{$\begin{array}{l}\text { Bacterial strain/ } \\
\text { Inoculation } \\
\text { mode }\end{array}$} & \multicolumn{3}{|c|}{ Day 7} & \multicolumn{3}{|c|}{ Day 9} & \multicolumn{3}{|c|}{ Day 16} \\
\hline & & no. & $g / b$ & $\mathrm{~g} / \mathrm{m}$ & no. & $\mathrm{g} / \mathrm{b}$ & $\mathrm{g} / \mathrm{m}$ & no. & $\mathbf{g} / \mathbf{b}$ & $\mathrm{g} / \mathrm{m}$ \\
\hline 1. & $\begin{array}{l}P . \text { aeruginosa } \\
247 / 1464 \text { i.p. }\end{array}$ & 4 & 130 & 32.5 & 4 & 140 & 35 & 4 & 149 & 37.25 \\
\hline 2. & $\begin{array}{l}\text { P. aeruginosa } \\
247 / 1464 \text { i.v. }\end{array}$ & 4 & 135 & 33.75 & 4 & 143 & 35.75 & 4 & 148 & 37 \\
\hline 3. & $\begin{array}{l}\text { P. aeruginosa } \\
247 / 1464 \text { i.n. }\end{array}$ & 4 & 141 & 35.25 & 4 & 146 & 36.5 & 4 & 150 & 37.5 \\
\hline 4. & $\begin{array}{l}\text { P. aeruginosa } \\
247 / 1464 \text { p.o. }\end{array}$ & 4 & 143 & 35.75 & 4 & 155 & 38.75 & 4 & 158 & 39.5 \\
\hline 5. & $\begin{array}{l}\text { P. aeruginosa } \\
492 \text { i.p. }\end{array}$ & 4 & 131 & 32.75 & 4 & 145 & 36.25 & 4 & 147 & 36.75 \\
\hline 6. & $\begin{array}{l}\text { P. aeruginosa } \\
492 \text { i.v. }\end{array}$ & 4 & 129 & 32.25 & 4 & 138 & 34.5 & 4 & 144 & 36 \\
\hline 7. & $\begin{array}{l}\text { P. aeruginosa } \\
492 \text { i.n. }\end{array}$ & 4 & 136 & 34 & 4 & 142 & 35,5 & 4 & 147 & 36.75 \\
\hline 8. & $\begin{array}{l}\text { P. aeruginosa } \\
492 \text { p.o. }\end{array}$ & 4 & 139 & 34.75 & 4 & 147 & 36.75 & 4 & 152 & 38 \\
\hline 9. & $\begin{array}{l}\text { P. aeruginosa } \\
37 \quad \text { i.p. }\end{array}$ & 4 & 120 & 30 & 4 & 124 & 31 & 4 & 146 & 36.5 \\
\hline 10. & $\begin{array}{l}\text { P. aeruginosa } \\
37 \quad \text { i.v. } \\
\end{array}$ & 4 & 122 & 30.5 & 4 & 145 & 36.25 & 4 & 152 & 38 \\
\hline 11. & $\begin{array}{l}\text { P. aeruginosa } \\
37 \quad \text { i.n. } \\
\end{array}$ & 4 & 122 & 30.5 & 4 & 137 & 34.25 & 4 & 142 & 35.5 \\
\hline 12. & $\begin{array}{l}\text { Ps. aeruginosa } \\
37 \quad \text { p.o. }\end{array}$ & 4 & 135 & 33.75 & 4 & 144 & 36 & 4 & 148 & 37 \\
\hline 13. & $\begin{array}{l}\text { P. aeruginosa } \\
1489 \text { i.p. }\end{array}$ & 3 & 72 & 24 & 2 & 58 & 29 & 2 & 62 & 31 \\
\hline 14. & $\begin{array}{l}\text { P. aeruginosa } \\
1489 \text { i.v. }\end{array}$ & 4 & 120 & 30 & 4 & 148 & 37 & 4 & 150 & 37.5 \\
\hline 15. & $\begin{array}{l}\text { P. aeruginosa } \\
1489 \text { i.n. }\end{array}$ & 4 & 119 & 29.75 & 3 & 92 & 30.6 & 3 & 105 & 35 \\
\hline 16. & $\begin{array}{l}\text { P. aeruginosa } \\
1489 \text { p.o. }\end{array}$ & 4 & 125 & 31.25 & 4 & 122 & 30.5 & 4 & 137 & 34.25 \\
\hline 17. & Control & 5 & 124 & 24.8 & 5 & 143 & 28.6 & 5 & 148 & 29.6 \\
\hline
\end{tabular}

no. $=$ number of mice/batch; $\mathrm{g} / \mathrm{b}=$ gram $/ \mathrm{batch} ; \mathrm{g} / \mathrm{m}=$ gram $/$ mouse $;-=$ animals died during the experiment 
Table 3. Viable cell counts $(\mathrm{CFU} / \mathrm{ml})$ in internal organ tissues of animal batches infected with untreated (PLA-) and PLA treated (PLA+) P. aeruginosa cultures.

\begin{tabular}{|c|c|c|c|c|c|c|c|c|c|}
\hline \multirow[t]{2}{*}{ No. } & \multirow{2}{*}{$\begin{array}{l}\text { Bacterial strain/ } \\
\text { Inoculation route }\end{array}$} & \multicolumn{2}{|c|}{$\begin{array}{l}\text { CFU/ml } \\
\text { dil. } 10^{-6} \\
\end{array}$} & \multicolumn{2}{|c|}{$\begin{array}{l}\text { CFU/ml } \\
\text { dil. } 10^{-7}\end{array}$} & \multicolumn{2}{|c|}{$\begin{array}{l}\text { CFU/ml } \\
\text { dil. } 10^{-8} \\
\end{array}$} & \multicolumn{2}{|c|}{$\begin{array}{l}\text { CFU/ml } \\
\text { dil. } 10^{-9} \\
\end{array}$} \\
\hline & & PLA- & PLA+ & PLA- & PLA+ & PLA- & PLA+ & PLA- & PLA+ \\
\hline \multirow[t]{5}{*}{1} & P. aeruginosa 1489 i.p. & & & & & & & & \\
\hline & Gall bladder & $\mathrm{NC}$ & - & 300 & - & 91 & - & 5 & - \\
\hline & Lung & $\mathrm{NC}$ & 61 & 460 & 17 & 80 & 9 & 30 & - \\
\hline & Intestine & 100 & 20 & 31 & - & - & - & - & - \\
\hline & Blood & $\mathrm{NC}$ & - & $\mathrm{NC}$ & - & $\mathrm{NC}$ & - & $\mathrm{NC}$ & - \\
\hline \multirow[t]{5}{*}{2} & P. aeruginosa 247/1464 i.p. & & & & & & & & \\
\hline & Gall bladder & $\mathrm{NC}$ & - & 173 & - & 95 & - & 10 & - \\
\hline & Lung & $\mathrm{NC}$ & - & $\mathrm{NC}$ & - & 121 & - & 28 & - \\
\hline & Intestine & $\mathrm{NC}$ & - & $\mathrm{NC}$ & - & 150 & - & 60 & - \\
\hline & Blood & $\mathrm{NC}$ & - & $\mathrm{NC}$ & - & $\mathrm{NC}$ & - & $\mathrm{NC}$ & - \\
\hline \multirow[t]{5}{*}{3} & P. aeruginosa 247/1464 i.v. & & & & & & & & \\
\hline & Gall bladder & 107 & - & 20 & - & - & - & - & - \\
\hline & Lung & 241 & - & 64 & - & 10 & - & - & - \\
\hline & Intestine & 26 & - & - & - & - & - & - & - \\
\hline & Blood & $\mathrm{NC}$ & - & $\mathrm{NC}$ & - & $\mathrm{NC}$ & - & $\mathrm{NC}$ & - \\
\hline \multirow[t]{5}{*}{4} & P. aeruginosa 492 i.v. & & & & & & & & \\
\hline & Gall bladder & $\mathrm{NC}$ & - & $\mathrm{NC}$ & - & 130 & - & 80 & - \\
\hline & Lung & 300 & - & 96 & - & 42 & - & 4 & - \\
\hline & Intestine & $\mathrm{NC}$ & - & $\mathrm{NC}$ & - & 50 & - & 30 & - \\
\hline & Blood & $\mathrm{NC}$ & - & 234 & - & 67 & - & 7 & - \\
\hline \multirow[t]{5}{*}{5} & P. aeruginosa 492 i.v. & & & & & & & & \\
\hline & Gall bladder & $\mathrm{NC}$ & - & $\mathrm{NC}$ & - & $\mathrm{NC}$ & - & $\mathrm{NC}$ & - \\
\hline & Lung & $\mathrm{NC}$ & - & $\mathrm{NC}$ & - & 27 & - & 2 & - \\
\hline & Intestine & $\mathrm{NC}$ & - & $\mathrm{NC}$ & - & $\mathrm{NC}$ & - & $\mathrm{NC}$ & - \\
\hline & Blood & - & - & - & - & - & - & - & - \\
\hline \multirow[t]{5}{*}{6} & P. aeruginosa 37 i.p. & & & & & & & & \\
\hline & Gall bladder & - & - & - & - & - & - & - & - \\
\hline & Lung & - & - & - & - & - & - & - & - \\
\hline & Intestine & - & - & - & - & - & - & - & - \\
\hline & Blood & - & - & - & - & - & - & - & - \\
\hline
\end{tabular}


Table 3. Cont.

\begin{tabular}{|c|c|c|c|c|c|c|c|c|c|}
\hline \multirow[t]{2}{*}{ No } & \multirow[t]{2}{*}{$\begin{array}{l}\text { Bacterial strain, inoculation } \\
\text { route }\end{array}$} & \multicolumn{2}{|c|}{$\begin{array}{l}\text { CFU/ml } \\
\text { dil. } 10^{-6}\end{array}$} & \multicolumn{2}{|c|}{$\begin{array}{l}\text { CFU/ml } \\
\text { dil. } 10^{-7}\end{array}$} & \multicolumn{2}{|c|}{$\begin{array}{l}\text { CFU/ml } \\
\text { dil. } 10^{-8}\end{array}$} & \multicolumn{2}{|c|}{$\begin{array}{l}\text { CFU/ml } \\
\text { dil. } 10^{-9}\end{array}$} \\
\hline & & PLA- & PLA+ & PLA- & PLA+ & PLA- & PLA+ & PLA- & PLA+ \\
\hline \multirow[t]{5}{*}{7} & P. aeruginosa 37 i.v. & & & & & & & & \\
\hline & Gall bladder & $\mathrm{NC}$ & - & $\mathrm{NC}$ & - & $\mathrm{NC}$ & - & $\mathrm{NC}$ & - \\
\hline & Lung & $\mathrm{NC}$ & - & $\mathrm{NC}$ & - & $\mathrm{NC}$ & - & $\mathrm{NC}$ & - \\
\hline & Intestine & 10 & - & - & - & - & - & - & - \\
\hline & Blood & - & - & - & - & - & - & - & - \\
\hline \multirow[t]{5}{*}{8} & P. aeruginosa 1489 i.p. & & & & & & & & \\
\hline & Gall bladder & 127 & - & 21 & - & - & - & - & - \\
\hline & Lung & $\mathrm{NC}$ & - & $\mathrm{NC}$ & - & 260 & - & 96 & - \\
\hline & Intestine & $\mathrm{NC}$ & - & $\mathrm{NC}$ & - & - & - & - & - \\
\hline & Blood & $\mathrm{NC}$ & - & $\mathrm{NC}$ & - & $\mathrm{NN}$ & - & $\mathrm{NC}$ & - \\
\hline \multirow[t]{5}{*}{9} & P. aeruginosa 1489 i.v. & & & & & & & & \\
\hline & Gall bladder & $\mathrm{NC}$ & 356 & 186 & 110 & 62 & 23 & 6 & - \\
\hline & Lung & $\mathrm{NC}$ & - & $\mathrm{NC}$ & - & $\mathrm{NC}$ & - & $\mathrm{NC}$ & - \\
\hline & Intestine & 108 & - & 36 & - & - & - & - & - \\
\hline & Blood & - & - & - & - & - & - & - & - \\
\hline
\end{tabular}

NC- not countable at the chosen dilution

For opportunistic bacteria, such as Pseudomonas aeruginosa, the outcome of the complex bacterial cell-host interactions depend on cellular density [3]. Our in vivo experimental study using a holoxenic mice infection model showed that subinhibitory concentrations of PLA could act as a potent inhibitor of $P$. aeruginosa pathogenicity, without affecting the bacterial cells viability, this antagonistic effect depending on the infection route and the microbial tested strain.

\section{Conclusions}

The present study has proved the role of subinhibitory concentrations of PLA in the attenuation of $P$. aeruginosa virulence and pathogenicity, using an in vivo holoxenic mouse infection experimental model. Our results are accounting for the hypothesis that subinhibitory concentrations of PLA, which are not acting by affecting the bacterial cell viability, are probably interferring with the intracellular communication and the sequential and coordinated expression of different virulence factors, altering the success of this pathogen in the colonization of a sensitive host and the development of an infectious process. 


\section{Experimental}

\section{General}

The pathogenic Ps. aeruginosa strains used in our study were isolated in 2006 from invasive infections and identified comparatively by classical biochemical reactions and API 20NE microtests (BioMérieux). D-3-phenyl lactic acid, purity $\geq 97.0 \%$ (PLA, Figure 1) supplied by Sigma-Aldrich Division (Milan, Italy) was used in the study.

Figure 1. $D$-3-phenyllactic acid.<smiles>O=C(O)[C@H](O)Cc1ccccc1</smiles>

Conventional (holoxenic) mice, purchased from Darvari Farm (Bucharest), were used in these experiments. In order to obtain comparative results the animals were grouped in different batches of five animals each as follows: one negative control (batch 1) and six infection batches and six batches for investigation of the influence of PLA on bacterial pathogenicity. Each batch contained 5 animals.

\section{Study design}

The bacterial strains were seeded on agar medium and incubated for 24 hours at $37{ }^{\circ} \mathrm{C}$. Starting from isolated colonies developed on the agar medium, microbial suspensions of 0.5. MacFarland density $\left(1-3 \times 10^{8} \mathrm{CFU} / \mathrm{ml}\right)$ were prepared in sterile saline. $100 \mu \mathrm{l}$ of this inoculum were distributed in two culture tubes for each strain, one containing $10 \mathrm{ml}$ nutrient broth and the other one $10 \mathrm{ml}$ nutrient broth with $D$-3-phenyl lactic acid at a final concentration of $1 \mathrm{mg} / \mathrm{ml}$. The concentration of PLA used in this study $(1 \mathrm{mg} / \mathrm{ml})$ proved to be subinhibitory for all tested strains, meaning that it did not affect the cultures viability, as demonstrated by the viable cell counts (CFU/ml) performed on the inoculum giving similar values in the presence as well as in the absence of $D$-3-phenyllactic acid. The initial solution of $100 \mathrm{mg} / \mathrm{ml}$ was prepared in vol:vol methanol:water solution. The solution was sterilised by $22 \mu \mathrm{m}$ filter mambrane. After adding the $D$-3-phenyllactic acid in the nutrient broth, the $\mathrm{pH}$ medium was controlled (in order to exclude the medium acidification due to the addition of the PLA). The two tubes were incubated for 24 hours at $37{ }^{\circ} \mathrm{C}$. The obtained overnight cultures of $P$. aeruginosa grown with and without addition of subinhibitory concentrations of PLA $(1 \mathrm{mg} / \mathrm{ml})$ were centrifuged and the sediment was washed three times in PBS (phosphate buffered saline). Starting from the cell sediment, bacterial suspensions of $\sim 10^{7} \mathrm{CFU} / \mathrm{ml}$ were prepared in PBS and used for mice inoculation in one single dose given by oral (p.o.), intranasal (i.n.), intravenous (i.v.), and intraperitoneal (i.p.) routes. The mice were followed up to 16 days after infection and the body weight, mortality and morbidity rate were measured at different time intervals. The course of infection was determined by daily examination of the animals in each batch, as well as by final viable cell count (Colony Forming Units, CFU number) performed on different organs and tissues, in order to appreciate the pathogen invasion (lungs, spleen, intestinal mucosa and blood). The tissue specimens (collected after the animals died from infection or were euthanized) were weighted and homogenised in sterile PBS. Serial ten-fold 
dilutions from homogenate were plated $(0.1 \mathrm{ml} / \mathrm{plate})$ onto King A medium specific for the isolation and counting of $P$. aeruginosa. The seeded media were incubated at $37{ }^{\circ} \mathrm{C}$ for 24 hours and the specific $P$. aeruginosa colonies were counted and the results were expressed in CFU/ml.

\section{References}

1. Suga H., Smith, K.M. Molecular Mechanisms of Bacterial Quorum Sensing as a New Drug Target. Curr. Op. Chem. Biol. 2003, 586-591.

2. Donlan, R.M., Costerton, J.W. Biofilms: Survival Mechanisms of Clinically Relevant Microorganisms. Clin. Microbiol. Rev. 2002, 15, 167-193.

3. Pearson, J.P.; Feldman, M.; Iglewski, B.H.; Prince, A. Pseudomonas Aeruginosa Cell to Cell Signaling Is Required for Virulence in a Model of Acute Pulmonary Infection. Infect. Immun. 2000, 68, 4331-4334.

4. Kiewit, T.R.; Iglewski, B.H. Bacterial Quorum-Sensing in Pathogenic Relationship. Infect. Immun. 2000, 68, 4839-4859.

5. Venturi, V. Regulation Of Quorum Sensing in Pseudomonas. FEMS Microbiol. Rev. 2006, 30, 274-291.

6. Hentzer, M.; Givskov, M. Pharmacological Inhibition of Quorum Sensing for the Treatment of Chronical Infections. J. Clin. Invest. 2003, 112, 1300-1307.

7. Valerio, F.; Lavermicocca, P.; Pascale, M.; Visconti, A., Production of Phenyllactic Acid by Lactic Acid Bacteria: an Approach to the Selection of Strains Contributing to Food Quality and Preservation. FEMS Microbiol. Lett. 2004, 233, 289-295.

8. Lazar, V.; Balotescu, M. C.; Vassu, T.; Barbu, V.; Smarandache, D.; Sasarman, E.; Israil A.; Bulai, D.; Alexandru, I.; Cernat, R. Experimental Study on Rats of the Probiotic Effect of Some Lactic Acid Bacteria Previously Selected tor Their In Vitro Capacity to Interfere with Salmonella Enteritidis Infection. Roum. Biotech. Lett. 2005, 10, 2123 - 2133.

9. Hentzer, M.; Wu, H.; Andersen, J.B.; Riedel, K.; Rasmussen, T.B.; Bagge, N.; Kumar, N.; Schembri, M.; Song, Z.; Kristoffersen, P.; Manefield, M.; Costerton, J.W.; Molin, S.; Eberl, L.; Steinberg, P.; Kjelleberg, S.; Høiby, N.; Givskov, M. Attenuation of Pseudomonas Aeruginosa Virulence by Quorum Sensing Inhibitors. EMBO J. 2003, 22, 3803- 3815.

10. Marco, M.L.; Legac, J; Lindow, S.E. Pseudomonas Syringae Genes Induced During Colonization Of Leaf Surfaces. Environ. Microbiol. 2005, 7, 1379-1391

(C) 2007 by MDPI (http://www.mdpi.org). Reproduction is permitted for noncommercial purposes. 